IAT and Pavlovian Conditioning 1

Running head: IAT and Pavlovian Conditioning

Changes in implicit associations do not occur simultaneously to Pavlovian conditioning of physiological anxiety responses.

Mark J. Boschen PhD, Innes Parker BPsych \& David L. Neumann PhD School of Psychology, Griffith University

KEYWORDS: Implicit associations, phobia, conditioning, anxiety disorders, anxiety

* Requests for reprints should be addressed to Dr Mark Boschen, School of Psychology, Griffith University, PMB 50 Gold Coast Mail Centre, Queensland 9726, Australia. Email: m.boschen@griffith.edu.au, Phone: +61 7 5552-8283, Fax +61 7 5552-8291 


\begin{abstract}
Previous research shows that an 'angry face' conditioned stimulus will elicit conditioned skin conductance responses (SCR) after a small number of trials pairing it with a mild electric shock. Such conditioning occurs even with masked presentations of the facial stimulus. Furthermore, the Implicit Association Test (IAT) has been demonstrated to be sensitive to the presence of information processing biases seen in specific phobias. We examined the ability of the IAT to detect changes in implicit associations that arise from pairing one facial image $(\mathrm{CS}+)$ with a mild electrotactile stimulation whereas a second facial image (CS-) was presented alone. Participants (N $=117)$ were assigned to one of four groups: A group that received no shocks with unmasked presentation of the facial images; a group that received no shocks with masked presentation; a group with shocks delivered simultaneously to the masked presentation of the $\mathrm{CS}+$; and a group that received shocks simultaneous to unmasked CS+ presentation. Learning was assessed through both SCR and the IAT. Results showed that while it was possible to condition a SCR to the $\mathrm{CS}+$, this learning was not detectable using the IAT. Implications of the discrepancies in the outcome measures are discussed.
\end{abstract}


Changes in implicit associations do not occur simultaneously to Pavlovian conditioning of physiological anxiety responses.

Anxiety Disorders are the single most prevalent cluster of psychological disorders in the general population (Mineka \& Zinbarg, 2006), resulting in significant distress and impairment to individual sufferers (e.g. Rapaport, Clary, Fayyad \& Endicott, 2005). Despite recent advances in our understanding of the aetiology of anxiety disorders, the exact mechanisms of the acquisition of anxiety responses are not completely understood. The acquisition of phobias is thought to occur through three (Rachman, 1978, 1990) or four (Lovibond, 2001) mechanisms, including Pavlovian conditioning. The ability of Pavlovian conditioning methods to elicit phobic symptoms in individuals has been repeatedly established over the past century of behavioral research in animals and humans (e.g. Watson \& Raynor, 1920; Rachman, 1991).

Anxiety and phobia present with both physiological responses to threat stimuli, as well as measurable changes in the processing of threat-related information. Physiological responses include measures of general arousal such as accelerated heart rate and increased skin conductance (Yartz \& Hawk, 2001; Hohen-Saric \& McLeod, 2000). Information processing changes include attentional biases to threat information (e.g. Mogg, Bradley, Miles \& Dixon, 2004; Mogg, Mathews \& Eysenck, 1992), increased dwell-time on threat stimuli (e.g. Koster, Crombez, Verschuere \& De Houwer, 2004; Fox, Russo \& Dutton, 2002; Fox, Russo, Bowles \& Dutton, 2001), and vulnerability to Stroop-type interference effects (e.g. Williams, Mathews \& McLeod, 1996; de Ruiter \& Brosschot, 1994; Mogg, Kentish \& Bradley, 1993). 
Despite knowledge of both behavioral and cognitive changes that accompany phobia acquisition, several components of the relationship between the behavioral and cognitive changes remain unclear. Firstly, Pavlovian conditioning paradigms are readily able to result in the acquisition of anxiety responses, such as increases in autonomic arousal, to a wide array of stimuli, particularly those that have evolutionary value (e.g. Öhman \& Mineka, 2001). However, the ability of such paradigms to also generate the information processing biases typically seen in anxiety disorders is not yet clear.

Secondly, if such changes in information processing occur through Pavlovian conditioning, it remains unclear whether autonomic physiological responses and information-processing artefacts develop in parallel or at different stages in the acquisition process. If information-processing biases develop in parallel with the behavioral acquisition of a conditioned response, this may suggest a single underlying mechanism, or mutually influential systems. Alternatively, if the development of cognitive biases and the conditioned anxiety response do not develop in parallel, this would suggest independent systems are responsible for the behavioral and information processing effects of anxiety disorders. Such a result may also suggest that the changes that occur as a result of conditioning are a precursor, or even foundation, for the development of biases in cognition.

Thirdly, while it is known that Pavlovian conditioning can occur without conscious awareness of the threat stimulus (e.g. Esteves, Dimberg, \& Öhman, 1994; Olsson \& Phelps, 2004), it in not clear whether information-processing artefacts would be evident in people subjected to unconscious Pavlovian conditioning processes. Again, this has important implications for understanding the etiology of anxiety disorders. In the event that information processing biases develop in the 
absence of conscious awareness of a conditioned phobic response, this would further support the primacy of conditioning in the development of anxiety. It would also suggest that cognitive changes can occur in the absence of conscious awareness of such anxiety-relevant information and experience.

\section{The Implicit Association Test}

The Implicit Association Test (IAT; Greenwald, McGhee, \& Schwartz, 1998) is one method of assessing the existence of associations in a conceptual network. It consists of a computerized task where participants are required to sort a series of stimuli (words or pictures) into one of several provided categories. A series of stimuli such as 'Spaniel', 'Terrier', 'Tabby', and 'Siamese' may be presented, for example, with the participant sorting the words into categories such as 'Dogs' and 'Cats'. In the IAT-Anxiety, the categories themselves are also paired with concepts relating to anxiety such as 'Calm' and 'Fear'. In trials where the participant is matching words to category pairs that they associate (e.g. 'Dogs' and 'Fear' in a dog-phobic individual), response times are significantly faster than when matching unassociated pairs (e.g. 'Dogs' and 'Calm' in the same dog phobic individual). These rapid response times are thought to reflect the existing associations between the fearstimuli, and the concept/experience of fear (Teachman, Gregg \& Woody, 2001). The IAT has been shown to be sensitive to anxiety and to the information processing distortions seen in phobic disorders (Egloff \& Schmuckle, 2002). Using a sample of individuals with spider or snake phobia, Teachman, Gregg \& Woody (2001) showed that the IAT was capable of discriminating between the two phobic groups. Furthermore, the test-retest stability of the IAT in assessing anxiety has been demonstrated over periods of one week, one month, and one year (Egloff, Schwerdtfeger, \& Schmuckle, 2005). 
One potential explanation for the sensitivity of the IAT to the presence of anxiety-related information processing biases emerges from connectionist models, such as that of Foa \& Kozak (1986) and more recently Tryon (2005). In these models concepts, emotions, and behavioral responses are described as nodes in a complex connected network. Phobic disorders are represented in such models by numerous strong connections of a broad array of anxiety-related concepts with nodes representing fear/anxiety and avoidance behavior. For example, an individual with spider phobia would have strong connections between concepts that are spiderrelevant (e.g. spiders, small hairy animals, etc.) and anxious emotion nodes. The range of different stimuli which were connected within the fear structure may be particularly broad in phobic individuals, and very little activation of any node the fear structure may be required to activate the entire network, including the associated behavioral responses. The more rapid IAT response times may be indicative of stronger associations or more rapid activations in the individual's conceptual network.

The IAT is particularly suitable for the current study for several reasons. Firstly, it is known to be sensitive to the information processing biases seen in phobic disorders (e.g. Teachman, Gregg \& Woody, 2001). Secondly, the IAT is able to measure associations between concepts in cases where a person is not consciously aware of the presence of such associations. It is therefore suitable to assessing cognitive changes that may have occurred as a result of masked (i.e. unconscious, masked) conditioning procedures. Thirdly, as the IAT is based on subtle differences in response times, it is difficult for participants to consciously distort their results (Egloff \& Schmuckle, 2002).

The Current Study 
The present research examined the ability of an aversive Pavlovian conditioning task to effect changes in an individual's associative network, in the absence of conscious awareness of such conditioning. Such research will enable better elucidation of the interaction between conditioned anxiety responses and information processing changes in phobic anxiety. A differential conditioning procedure was used in which participants were exposed to images of two angry faces. Half the participants experienced a mild electro-tactile stimulus when one face (the $\mathrm{CS}+$ ) was displayed and no electrotactile stimulus when the other face (the CS-) was displayed. The remaining participants did not receive any electrotactile stimulus, but served as a control for conditioning and masking effects. Half the participants in each conditioning group were shown the facial stimuli masked in such a way as to prevent conscious awareness, whereas the remaining participants received unmasked presentations. The full factorial design is illustrated in Figure 1. Outcome was measured via two methods. The acquisition of a conditioned response to the $\mathrm{CS}+$ was assessed via the skin conductance response. The development of cognitive associations between the CS+ and the concept of anxiety/fear was assessed via the IAT.

The following hypotheses were tested: Firstly, we expected that participants who were exposed to the electro-tactile stimulus simultaneously with the CS+ would show greater skin conductance responses than those who did not receive such pairing, demonstrating the acquisition of fear conditioning. Moreover, the development of conditioned responses were expected to occur for both masked and unmasked CS+ presentations. Secondly, we expected the same pattern of significant effects when IAT responses were used as the DV. Such a result would suggest that behavioral acquisition of conditioned fear responses is accompanied by immediate changes in 
IAT and Pavlovian Conditioning 8

implicit associations. Alternatively, if implicit associations fail to develop in spite of successful conditioning, this would suggest independent pathways via which conditioning and implicit associations develop. 
Method

\section{Participants}

A total of 117 undergraduate psychology students participated in exchange for partial course credit. Two participants were excluded on the basis that they recognized the initial picture stimulus before it was masked (and therefore explicit conditioning could not be ruled out). A third participant was excluded because the skin conductance measures did not register more than half of the participant's responses. The final sample consisted of 114 participants (93 female, 21 male) with a mean age of 22.94 years $(S D=7.19)$ and a mean total length of formal education of 12.50 years $(S D=2.31)$. Participants were randomly allocated one of four groups such that there was a similar number of males and females in each group.

\section{Materials and Apparatus}

Materials and apparatus consisted of the experimental stimuli, pen-and-paper questionnaires, psychophysiological recording apparatus, and a test of implicit associations.

Facial Stimuli (NimStim Faces Set; Tottenham, Borscheid, Ellertsen, Marcus, \& Nelson, 2002; Tottenham, et al., 2006; Research Network on Early Experience and Brain Development, 2006). The NimStim Faces Set is a standard set of facial images, with differing emotional expressions. Three facial images (of different individuals) were chosen from the overall set: two with angry facial expressions, and one with a neutral facial expression. For the $\mathrm{CS}+$, the image $22 m \_a n \_o . B M P$ was used, while 26m_an_o.BMP was used for the CS-. These images were named "John" and "Tom" respectively, so that participants could differentially refer to either image. For masking, the image $23 m \_n e \_c . B M P$ of a neutral facial image was used. Details of the procedure for use of the masking image are presented in the Procedure section below. 
Electrotactile stimulus. The Electro-tactile stimulus was delivered by an IWORX SI100 stimulus isolator, via two disposable ADInstruments MLA1010B $\mathrm{Ag} / \mathrm{AgCl}$ electrodes, attached to the wrist of the participant's preferred forearm. The stimulus isolator functions as a constant current device that will output the voltage necessary to maintain the set current flow, up to a maximum of $100 \mathrm{~V}$. The maximum current that can be set is $10 \mathrm{~mA}$

Beck Anxiety Inventory (BAI; Beck, Epstein, Brown \& Steer, 1988). The BAI is a 21-item self-report questionnaire designed to measure arousal symptoms of anxiety. It is brief, commonly used, and psychometrically sound (Beck et al., 1988; Roemer, 2001; Osman, Kopper, Barrios, Osman, \& Wade, 1997).

Initial Response to Faces Questionnaire. A brief questionnaire assessed any pre-existing emotional reactions to the facial stimuli. The participant was given a sheet of paper with color images of the CS+ and CS-, and for each image responded to the question "How does this face make you feel?" on a linear scale ranging from 1 "It elicits negative feelings" to 7. "It elicits positive feelings".

Implicit Association Test (IAT; Greenwald et al., 1998). A customized version of the IAT assessed the development of implicit cognitive associations between the CSs and concepts of anxiety and threat. The IAT was administered on computer by using the DMDX experimental software package (Forster \& Forster, 2003). The IAT was presented in a series of trial blocks with the layout of the display screen shown in Figure 3. For each block, two categories (e.g. "Safety" and "Danger") were displayed at the top of the screen for one second prior to presentation of the stimulus. Participants were required to match the stimulus word/picture to the appropriate category using either the right or left Shift key. 
The IAT consists of six Blocks of stimuli, with each block presented in the same sequence for each participant. Block 1 was a practice block, consisting of 10 stimuli of blue and yellow circles, and the matching categories of "Blue" and "Yellow". Participants were shown one of the colored circle stimuli, and asked to match it to the appropriate word in the upper-left or upper-right of the screen using the left and right Shift keys. This practice block was designed to ensure that each participant understood the task, and was able to use the keyboard to match the stimuli with the color-concepts. Block 2 consisted of an assessment of the participant's recognition of the names assigned to the CS+ and CS-. A series of twenty trials, with ten presentations each of the CS+ and CS- were run, with the participant being required to match to the concepts "John" and "Tom". Block 3 was designed to assess the participants' comprehension of the stimulus words, through their ability to accurately match words to concepts of "Safety" and "Danger". In Block 3, a total of 20 trials were run, with each stimulus word presented twice. The order of presentation was scrambled. The word list of Danger-related and Safety-related words used in Block 3 is reproduced in the Appendix.

IAT Block 4 introduced matching to two simultaneous, congruent categories. In this block, the words "Tom" and "Safety" were presented in the upper-left of the screen, while the words "John" and "Danger" were displayed in the upper-right. Stimuli were presented for a total of 80 trials. The first set of 20 trials was used as a practice task, and timings were not used in subsequent analyses. The following 60 trials consisted of 5 Danger-related words (each displayed 3 times), 5 Safety-related words (each displayed 3 times), and the facial images of the CS+ and CS- (each displayed 15 times). 
Block 5 was identical to Block 3, except that the position of the categories "Danger" and "Safety" was reversed. Block 6 was identical to Block 4, except that the category pairings were incongruent, with "Danger" and "Tom" shown in the upper-left, and "Safety" and "John" displayed in the upper-right. A summary of the IAT trials used in the assessment of implicit associations is given in Table 1.

Psychophysiological Assessment. Skin conductance and respiration were recorded with a Dell Optiplex GX260 personal computer interfaced with a PowerLab Model 4/20 (ADInstruments, Sydney) physiological data acquisition system. Skin conductance was measured via an ADInstruments Model ML116 GSR amplifier and standard MLT116F electrodes attached to the distal phalanges of the first and second digits of the non-preferred hand. Respiration was monitored using an ADInstruments MLT1132 Piezo Respiratory belt transducer, attached around the chest, below the sternum. Skin conductance signals were acquired during the Acquisition and Assessment phases of the experiment using a sampling rate of $1000 \mathrm{~Hz}$ and stored offline for later quantification.

\section{Procedure}

The participant sat opposite to a computer monitor for the administration of conditioning and the IAT. The experimenter monitored the experiment via a closed circuit video system in an adjoining room. The mean temperature and humidity in the participant's room was $20.5^{\circ} \mathrm{C}$ and $57.25 \%$, respectively. Participants were randomly assigned to one of four groups. Half the participants were allocated to the masked presentation group (i.e. where the facial stimuli would be masked, preventing conscious awareness), while the other half received unmasked presentation of all facial stimuli (Mask vs NoMask). Half of the participants in each masking condition were exposed to electro-tactile stimulation simultaneously to presentation of one of 
the two faces (the $\mathrm{CS}+$ ), while the other half in each masking condition received no electrical stimulation (ETS vs NoETS). This yielded four experimental groups (MaskETS, Mask-NoETS, NoMask-ETS, and NoMask-NoETS). All participants proceeded through a series of five phases during the experiment. Only Phase III (the Acquisition Phase) differed between the four groups.

Screening and Work-Up Phase (Phase I). Each participant completed the standard series of questionnaires, comprized of the BAI and the Initial Response to Faces Questionnaire. All of these were administered as pen-and-paper measures. Following the questionnaires, participants were connected to the psychophysiological recording equipment, and each individual proceeded through a work-up procedure designed to identify a level of electro-tactile stimulation that would be experienced as 'unpleasant but not painful'. This final level was used for the remainder of the experiment.

Habituation Phase (Phase II). Participants were allowed to habituate to the experimental environment. During this phase, the CS+ and CS- were each presented three times in random order, for a total of six trials. The facial stimulus was displayed in conjunction with the name given to each image (i.e. "John" for the CS+ and "Tom" for the CS-). Each stimulus was displayed for $6 \mathrm{~s}$, with a $13 \mathrm{~s}$ intertrial interval. Following the stimulus presentations, participants rested for two minutes before beginning the next stage.

Acquisition Phase (Phase III). Phase III was completed differently for participants assigned to each condition. A series of twelve trials were conducted, in which the CS+ and CS- were presented six times each, in random order. In each presentation, the facial image stimulus was presented at the start of each trial (i.e. 0ms). For the participants in the Mask presentation conditions the CS+ and CS- were 
replaced by the masking facial image after $33 \mathrm{~ms}$, and was presented for a further 5967ms. For participants in the Unmasked presentation conditions, the CS+ and CSwere presented for $6000 \mathrm{~ms}$ and without any mask stimulus. For participants assigned to the electro-tactile stimulation (ETS) condition, the electro-tactile stimulus was delivered simultaneously with the onset of the CS+ (only) for $200 \mathrm{~ms}$. The interval between each trial was 13s. A graphic representation of the timing used in all four conditions is depicted in Figure 2.

For those participants exposed to masked presentations of the CS images, an initial check was made to check that they did not consciously recognise the faces, by showing each participant a paper image of the CS+ and CS- and asking if they recognised either from the previous experiment phase. Two participants reported such awareness, and were subsequently excluded from analysis.

Assessment Phase (Phase IV). Two assessments of fear acquisition were undertaken with all participants: skin conductance response measurement followed by a measure of implicit associations. Skin conductance responses provided a direct measure of Pavlovian conditioning. Skin conductance is commonly used as a valid and reliable method for measuring the physiological representations of learning from Pavlovian conditioning (e.g. Lovibond, 2003; Olsson \& Phelps, 2004). A total of 12 trials were used, with 6 presentations of the CS+ and 6 presentations of the CS-, all for a 10 s duration and delivered in random order. The phasic skin conductance responses elicited following the onset of the CS+ and CS- were measured. The amplitude of the response was measured within a latency window of 1 to $4 \mathrm{~s}$ following the onset of the CS. Responses associated with respiratory artefact (e.g., coughing, sneezing), as determined by visual inspection of the respiratory signal, were scored as missing. Following the measurement of skin conductance responses, the 
IAT was completed by all participants, as detailed above. Implicit associations were measured through the mean differences in latency of correct matches between Block 4 and Block 6.

Extinction Phase (Phase V). In Phase V, all participants completed a final extinction and debriefing process. Participants in all 4 experimental groups received 3 exposures each of the $\mathrm{CS}+$ and the CS-, each lasting 6s (with a 13s intertrial interval between exposures). They received these exposures in random order, without electrotactile stimulation or masking. Participants were verbally informed that no electrotactile stimulus would be administered. 


\section{Results}

\section{Equivalence of Anxiety across Groups}

A comparison of the baseline anxiety symptoms of each of the four experimental groups was conducted to ensure that no group had pre-existing elevations in anxiety that may distort the results. A one-way ANOVA with four levels (Mask-ETS, Mask-NoETS, NoMask-ETS, NoMask-NoETS) and BAI scores as the DV was conducted. The results indicated that all four groups were equivalent with respect to current anxiety symptoms $(F(3,110)=1.27, p>.05)$. Equivalence of Facial Stimuli

A paired samples $t$-test indicated that there prior to conditioning, participants felt significantly more positive towards the CS+ than the CS- $(t=4.60, d f=112, p<$ $\left..0005, M_{\mathrm{CS}+}=2.75, M_{\mathrm{CS}-}=2.28\right)$. This significant difference was not deemed problematic, as it was in the reverse direction to the proposed hypothesis that the CS+ would come to elicit negative reactions.

\section{Equivalence of Acquisition}

To ensure that acquisition of the conditioned response was equivalent in masked and unmasked conditions, a 2x6 ANOVA was conducted. The two IVs were Masking (Mask vs NoMask), and Trial (Trials 1 to 6, a within-subjects factor). The DV was the latency-to-peak amplitude difference from the measure of skin conductance for the CS+ presentations. The interaction between Masking and Trial was not significant, indicating that acquisition had been uniform across masked and unmasked participants $(F(1,114)=2.09, p>.05)$. The skin conductance responses from the Acquisition phase are presented in Figure 4.

Analysis of Variance - Skin Conductance 
To assess for the successful Pavlovian acquisition of an anxiety response to the CS+, a three-way ANOVA was conducted. The three IVs were Stimulus (CS+ vs CS-), Masking (Mask vs NoMask), and ETS (ETS vs NoETS,). The DV was the latency-to-peak amplitude difference from the measure of skin conductance. These results are shown graphically in Figure 5.

There was a significant simple effect of Stimulus on skin conductance recordings $\left(F(1,112)=110.83, p<.001, M_{\mathrm{CS}+}=1.39 \mu \mathrm{S}, M_{\mathrm{CS}-}=0.43 \mu \mathrm{S}\right)$. There was no significant difference on skin conductance recordings depending on whether masked or non-masked presentation was used $(F(1,112)=0.086, p>.05)$. The presence or absence of electro-tactile stimulation made a significant difference to overall skin conductance responses, with those receiving the electrical stimulus showing larger skin conductance changes $\left(F(1,112)=89.03, p<.001, M_{\mathrm{ETS}}=\right.$ $\left.1.57 \mu \mathrm{S}, M_{\mathrm{NoETS}}=0.25 \mu \mathrm{S}\right)$.

As expected, there was a significant interaction between Stimulus and ETS group $(F(1,112)=124.25, p<.001)$. As shown in Figure 5, skin conductance responses were larger during the CS+ than during the CS- only participants who received the electro-tactile stimulus contingent with $\mathrm{CS}+$ during the acquisition phase (Phase III). However, the use of masked CS presentations during acquisition did not influence conditioning, as shown by no significant interaction between Stimulus and Masking $(F(1,112)=0.35, p>.05)$, nor between ETS and Masking $(F(1,112)=$ $0.11, p>.05)$. The three-way interaction was also not significant $(F(1,112)=0.01, p$ $>.05)$.

Analysis of Variance - Implicit Association Test

To assess for the successful development of implicit associations between the $\mathrm{CS}+$ and danger concepts, a three-way ANOVA was conducted. The three IVs were 
Match (Consistent vs Inconsistent, a within-subjects IV), Masking (Mask vs NoMask, a between-subjects IV), and ETS (ETS vs NoETS, a between-subjects IV). The DV was the mean reaction time of correct IAT responses from Block 4 (consistent category matching) and Block 6 (inconsistent category matching). These results are shown graphically in Figure 6.

No main effects of the IVs were significant. There was no difference between reaction times to consistent and inconsistent categories $(F(1,111)=1.22, p>.05)$. There was no significant difference on reaction time depending on whether masked or non-masked presentation was used during the Acquisition Phase $(F(1,111)=0.97, p$ $>.05)$. The presence or absence of electro-tactile stimulation made no difference to mean reaction time $(F(1,111)=0.17, p>.05)$. Contrary to expectation, there was no significant interaction between Match and ETS group $(F(1,111)=1.36, p>.05)$. There was no significant interaction between Match and Masking $(F(1,111)=2.51, p$ $>.05)$, nor between ETS and Masking $(F(1,111)=0.58, p>.05)$. The three-way interaction was also not significant $(F(1,111)=0.16, p>.05)$. 


\section{Discussion}

This study aimed to assess the ability of a Pavlovian conditioning paradigm to rapidly lead to the development of implicit cognitive associations. The pairing of a CS+ with an unpleasant electrotactile stimulus was hypothesized to not only elicit a strong conditioned skin conductance response, but also to create implicit associations between the CS+ and the concept of danger. The results partially supported the hypotheses. The pairing of an angry face CS with an electro-tactile stimulus lead to significant conditioned skin conductance responses when compared with an angry face CS that was not paired with the electro-tactile stimulus (within-subjects comparison) or the same angry face that was not paired with the electro-tactile stimulus in another group of participants (between-groups comparison). The strength of the conditioned response that was observed suggested that as few as six pairings were adequate to for the association to be learnt. Importantly, the association was formed between the angry face CS and the electro-tactile stimulus even when the CS presentations were masked. However, despite the strong conditioning effects that were observed in the skin conductance response, the IAT did not reveal any significant differences in implicit associations between the two facial images. It is worth noting also, that whether the CS+ and CS- were presented for a perceptible period, or in a masked presentation method, had no effect on the implicit association measures.

It may be argued that the present conditioning paradigm or number of trials may have not been sufficiently powerful to cause changes in cognitive processing of the CS+/CS- as measured by the IAT. While conditioned skin conductance responses were reliably observed, changes in cognition may be delayed, or require further S-R pairing/experiences. The IAT may not be sufficiently sensitive to detect subtle 
changes in concept associations. Although the IAT has been demonstrated to be sensitive to the diagnosis of specific phobias, the less extreme fear conditioning of our research may not have been sufficient to elicit detectable changes in information processing.

An alternative explanation is that Pavlovian conditioning and development of cognitive associations occur via different pathways. This explanation is supported by research which argues that the amygdala and the hippocampus play different roles in fear (e.g. Mineka \& Öhman, 2002; Öhman \& Mineka, 2001). While the hippocampus operate at a cognitive level, processing through the amygdala is automatic, encapsulated, and not amenable to conscious control. The observed dissociation between the measures of skin conductance and IAT is to be expected if these measures assessed learning of different pathways.

Integration with Previous Research

Not surprisingly, our research replicates the ability of electro-dermal stimulation to elicit conditioned anxiety responses, even when the CS+ is presented in a masked manner. This is consistent with reports by Öhman, Flykt, \& Lundquist (2000) who also used angry facial expressions, reporting that these survive masking effects.

At first glance, the findings may appear inconsistent with those reported by Teachman et al. (2001) who reported that the IAT was able to detect implicit associations that were present in individuals with snake or spider phobias. The study by Teachman et al. used students with a measured difference between their selfreported snake and spider fear ratings. In this case, only individuals with existing fears were used. This contrasts with our participants who received new conditioning using a novel CS+/CS-. Connectionist models of anxiety would assert that phobias 
are significant and problematic because they consist of a network which the anxiety and fear nodes are easily activated by the phobic stimulus. In the case of our participants, it is almost certain that the associations between the CS+ and anxiety reaction were very specific, and had not yet generalized. Even so, this is significant, indicating that subtle changes in behavioral responses may be easier to elicit than changes in the broader network.

\section{Limitations of the Current Study}

Despite the interesting findings from this research, limitations must be considered when evaluating the results. Throughout the current study, the IAT was used as a measure of associations in a network of anxiety and related concepts. The assumption that the IAT is a valid measure of these network associations is based on a small set of studies which use the test in anxiety research. Further investigation of the suitability of the IAT to such measurement needs to be undertaken, and the findings of such research may eventually limit the findings presented here.

\section{Future Directions}

The findings from this research suggest several fruitful avenues for future investigation. These fall into two broad areas: the first of these pertains to the use of the IAT in future research, while the second entails further investigation of fear acquisition.

The current study used the IAT as an indirect measure of the cognitive associations held between anxiety and other concepts. While such use of the IAT is supported by previous research (e.g. Egloff \& Schmuckle, 2002; Teachman et al., 2001), there is considerable further work to demonstrate conclusively that the IAT is measuring the sort of network connections as proposed in models such as that of Foa \& Kozak (1986). Such research may examine the relationship between IAT measures 
and other measures of such associations such as Stroop-type tasks. Additional support for the IAT as a measure of cognitive associations would be gained through the attenuation of implicit associations following successful treatment.

Our results suggest that implicit associations are developed separately to the conditioned arousal response. Further research into the separate mechanisms by which implicit associations are acquired over time may assist in explaining these results. Furthermore, such investigations would shed further light on the methods by which Rachman's $(1978,1991)$ three pathways to anxiety operate, and whether each mechanism results in equally-rapid acquisition of anxiety responses.

With known individual differences in conditionability, it may also be true that some individuals develop implicit associations more rapidly than others. For example, individuals with high levels of anxiety sensitivity or neuroticism are known to be at increased risk of anxiety disorders. It may be expected, therefore, that such people may more rapidly or strongly acquire implicit associations between a wide array of concepts and the concept of anxiety/threat. Such a finding may assist in explaining the vulnerability of these individuals to anxiety problems.

\section{Conclusion}

The results presented here are offered as an initial attempt to investigate whether conditioned anxiety responses are immediately accompanied by the acquisition of implicit associations. Despite some limitations, these findings suggest that underlying associations in cognitive structures may occur only at some point later than the development of the basic S-R pairing. 
IAT and Pavlovian Conditioning 23 


\section{References}

Beck, A.T., Epstein, N., Brown, G., \& Steer, R.A. (1988). An inventory for measuring clinical anxiety: Psychometric properties. Journal of Consulting and Clinical Psychology, 56, 893-897.

de Ruiter, C. \& Brosschot, J.F. (1994). The emotional Stroop interference effect in anxiety: Attentional bias or cognitive avoidance? Behaviour Research and Therapy, 32, 315-319.

Egloff, B. \& Schmuckle, S.C. (2002). Predictive validity of an Implicit Association Test for assessing anxiety. Journal of Personality and Social Psychology, 83, 1441-1455.

Egloff, B., Schwerdtfeger, A., \& Schmuckle, S.C. (2005). Temporal stability of the Implicit Association Test - Anxiety. Journal of Personality Assessment, 84, $82-88$.

Esteves, F., Dimberg, U., \& Öhman, A. (1994). Automatically elicited fear: Conditioned skin conductance responses to masked facial expressions. Cognition and Emotion, 8, 393-413.

Foa, E.B. \& Kozak, M.J. (1986). Emotional processing of fear: Exposure to corrective information. Psychological Bulletin, 99, 20-35.

Forster, K.I. \& Forster, J.C. (2003). A Windows program with millisecond accuracy. Behaviour Research Methods, Instruments \& Computers, 35, 116-124.

Fox, E., Russo, R., Bowles, R., \& Dutton, K. (2001). Do threatening stimuli draw or hold visual attention in subclinical anxiety? Journal of Experimental Psychology: General, 130, 681-700. 
Fox, E., Russo, R., \& Dutton, K. (2002). Attentional bias for threat: Evidence for delayed disengagement from emotional faces. Cognition and Emotion, 16, 355-379.

Greenwald, A.G., McGhee, D.E., \& Schwartz, J.L K. (1998). Measuring individual differences in implicit cognition: The Implicit Association Test. Journal of Personality and Social Psychology, 74, 1464-1480.

Hohen-Saric, R. \& McLeod, D.R. (2000). Anxiety and arousal: Physiological changes and their perception. Journal of Affective Disorders, 61, 217-224.

Koster, E.H.W., Crombez, G., Verschuere, B., \& De Houwer, J. (2004). Selective attention to threat in the dot probe paradigm: Differentiating vigilance and difficulty to disengage. Behaviour Research and Therapy, 42, 1183-1192.

Lovibond, P.F. (2003). Casual beliefs and unconditional stimulus: Retrospective revaluation induced by experience and by instruction. Journal of Experimental Psychology: Learning, Memory and Cognition, 29, 97-106.

Mogg, K., Bradley, B.P., Miles, F., \& Dixon, R. (2004). Time course of attentional bias for threat scenes: Testing the vigilance-avoidance hypothesis. Cognition and Emotion, 18, 689-700.

Mogg, K., Kentish, J., \& Bradley, B.P. (1993). Effects of anxiety and awareness on colour-identification latencies for emotional words. Behaviour Research and Therapy, 31, 559-567.

Mogg, K., Mathews, A., \& Eysenck, M. (1992). Attentional bias to threat in clinical anxiety states. Cognition and Emotion, 6, 149-159.

Mineka, S. \& Öhman, A. (2002). Phobias and preparedness: The selective, automatic, and encapsulated nature of fear. Biological Psychiatry, 52, 927-937. 
Mineka, S., Watson, D., \& Clark, L.A. (1998). Comorbidity of anxiety and unipolar mood disorders. Annual Review of Psychology, 49, 377-412.

Olsson, A. \& Phelps, E. A., (2004). Learned fear of “unseen” faces after Pavlovian, observational, and instructed fear. Psychological Science, 15, 822- 828.

Öhman, A., Flykt, A., \& Lundquist, D., (2000). Unconscious emotion: Evolutionary perspectives, psychophysiological data, and neuropsychological mechanisms. In R. D. Lane \& L. Nadel (Eds.). The Cognitive Neuroscience of Emotion (pp. 296-327). New York: Oxford University Press.

Öhman, A. \& Mineka, S. (2001). Fears, phobias, and preparedness: Toward an evolved module of fear and fear learning. Psychological Review, 108, 483522.

Osman, A., Kopper, B.A., Barrios, F.X., Osman, J.R., \& Wade, T. (1997). The Beck Anxiety Inventory: Reexamination of factor structure and psychometrics properties. Journal of Clinical Psychology, 53, 7-14.

Rachman, S.J. (1978). Fear and Courage. W.H. Freeman and Company: San Francisco.

Rachman, S. (1991). Neo-conditioning and the classical theory of fear acquisition. Clinical Psychology Review, 11, 155-173.

Rapaport, M.H., Clary, C., Fayyad, R., \& Endicott, J. (2005). Quality-of-Life impairment in depressive and anxiety disorders. American Journal of Psychiatry, 162, 1171-1178.

Research Network on Early Experience and Brain Development (2006, November). NimStim Face Stimulus Set. Retrieved September 30, 2006, from the Research Network on Early Experience and Brain Development website: http://www.macbrain.org/resources.htm 
Roemer, L. (2001). Measures for anxiety and related constructs. In M.M. Antony, S.M. Orsillo, \& L. Roemer (eds.). Practitioners Guide to Empirically Based Measures of Anxiety. Kluwer Academic: New York.

Teachman, B.A., Gregg, A.P., \& Woody, S.R. (2001). Implicit associations for fearrelevant stimuli among individuals with snake and spider fears. Journal of Abnormal Psychology, 110, 226-235.

Tottenham, N., Borscheid, A., Ellertsen, K., Marcus, D.J., \& Nelson, C.A. (2002, April). Categorization of facial expressions in children and adults: Establishing a larger stimulus set. Poster presented at the Cognitive Neuroscience Society Annual Meeting, San Francisco.

Tottenham, N., Tanaka, J.W., Leon, A.C., McCarry, T., Nurse, M., Hare, T.A., et al. (2006). Validity and reliability of the NimStim set of facial expressions. Manuscript submitted for publication.

Tryon, W.W. (2005). Possible mechanisms for why desensitization and exposure therapy work. Clinical Psychology Review, 25, 67-95.

Watson, J.B. \& Raynor, R., (1920). Conditioned emotional reactions. Journal of Experimental Psychology, 3, 1-14.

Williams, J.M.G., Mathews, A., \& MacLeod, C. (1996). The emotional Stroop task and psychopathology. Psychological Bulletin, 120, 3-24.

Yarz, A.R. \& Hawk, L.W. (2001). Psychophysiological assessment of anxiety: Tales from the heart. In M.M. Antony, S.M. Orsillo, \& L. Roemer (eds.). Practitioners Guide to Empirically Based Measures of Anxiety. Kluwer Academic: New York. 
Author Note

Development of the NimStim Face Stimulus Set was overseen by Nim Tottenham and supported by the John D. and Catherine T. MacArthur Foundation Research Network on Early Experience and Brain Development. Please contact Nim Tottenham at tott0006@tc.umn.edu for more information concerning the stimulus set. 
Appendix - IAT Stimulus Words and Categories

\begin{tabular}{cc}
\hline Danger & Safety \\
\hline Panic & Calm \\
Terror & Safe \\
Threat & Protected \\
Pain & Peaceful \\
Evil & Good \\
\hline
\end{tabular}


Table 1

Summary of IAT Trials.

\begin{tabular}{|c|c|c|c|}
\hline \multirow{2}{*}{ Block } & \multicolumn{2}{|c|}{ Categories } & \multirow{2}{*}{$\begin{array}{c}\text { Stimulus Items } \\
\text { (Random Order within Blocks) }\end{array}$} \\
\hline & Left & Right & \\
\hline \multirow[t]{2}{*}{1} & Yellow & Blue & $5 \times$ Blue coloured circles \\
\hline & & & $5 \times$ Yellow coloured circles \\
\hline \multirow[t]{2}{*}{2} & Tom & John & 10 x Facial Image of CS+ (“John”) \\
\hline & & & 10 x Facial Image of CS- ("Tom") \\
\hline \multirow[t]{2}{*}{3} & Safety & Danger & 10 x Safety-Related Words \\
\hline & & & $10 \mathrm{x}$ Danger-Related Words \\
\hline \multicolumn{4}{|l|}{4} \\
\hline \multirow[t]{4}{*}{ (Practice) } & Tom & John & 5 x Safety-Related Words \\
\hline & Safety & Danger & 5 x Danger-Related Words \\
\hline & & & 5 x Facial Image of CS+ (“John”) \\
\hline & & & 5 x Facial Image of CS- (“Tom”) \\
\hline \multirow[t]{4}{*}{ (Assessed) } & Tom & John & 15 x Safety-Related Words \\
\hline & Safety & Danger & 15 x Danger-Related Words \\
\hline & & & 15 x Facial Image of CS+ (“John”) \\
\hline & & & 15 x Facial Image of CS- (“Tom") \\
\hline \multirow[t]{2}{*}{5} & Danger & Safety & 10 x Safety-Related Words \\
\hline & & & $10 \mathrm{x}$ Danger-Related Words \\
\hline \multirow{5}{*}{$\begin{array}{c}6 \\
\text { (Practice) }\end{array}$} & & & \\
\hline & Danger & Safety & 5 x Safety-Related Words \\
\hline & Tom & John & $5 \times$ Danger-Related Words \\
\hline & & & 5 x Facial Image of CS+ (“John”) \\
\hline & & & 5 x Facial Image of CS- (“Tom") \\
\hline \multirow[t]{4}{*}{ (Assessed) } & Danger & Safety & 15 x Safety-Related Words \\
\hline & Tom & John & 15 x Danger-Related Words \\
\hline & & & 15 x Facial Image of CS+ (“John”) \\
\hline & & & 15 x Facial Image of CS- (“Tom") \\
\hline
\end{tabular}




\section{Figure Captions}

Figure 1. 2x2x2 ANOVA Designs.

Figure 2. Timing for onset of facial stimuli, masking stimuli, and electro-tactile stimulation.

Figure 3. Sample IAT screen, with measurements expressed as percentages of the overall screen dimensions.

Figure 4. Skin conductance responses elicited by the CS+ over the six Acquisition trials for all groups.

Figure 5. Interaction between Stimulus image and ETS group on skin conductance responses for Masked and Unmasked presentations of the CSs.

Figure 6. Interaction between Stimulus image and ETS group on IAT response times for Masked and Unmasked presentations of the CSs. 

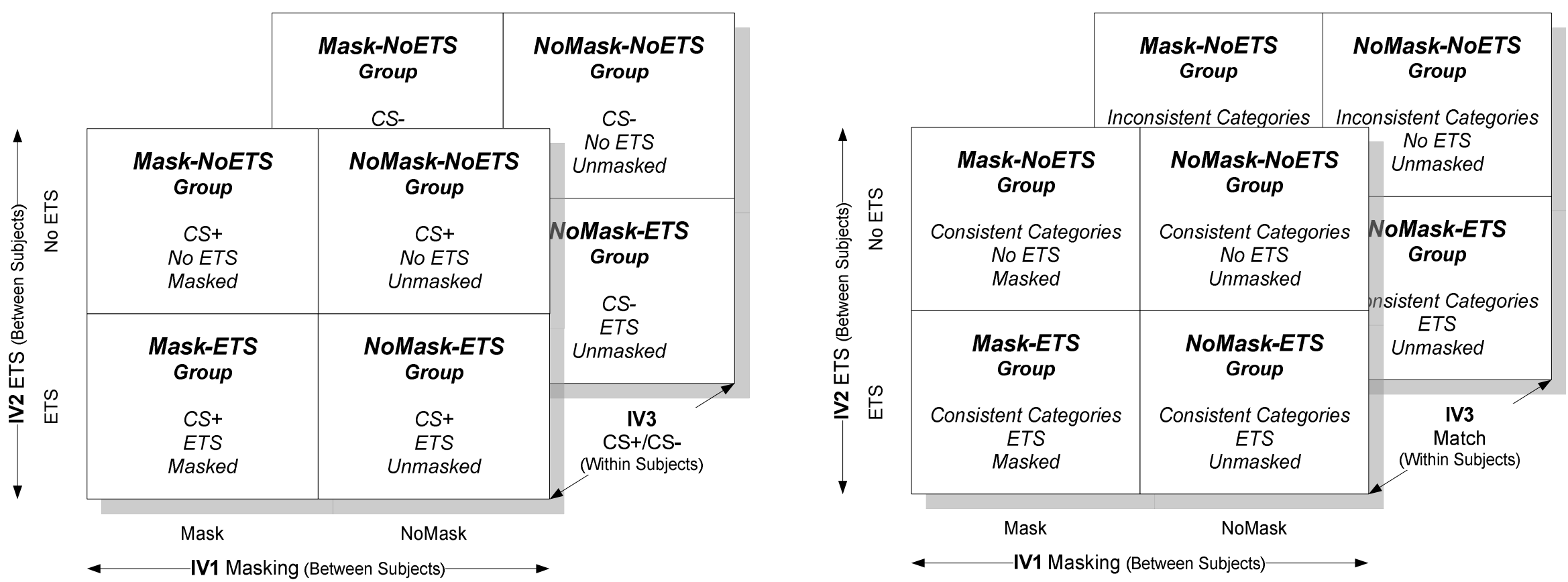
Mask

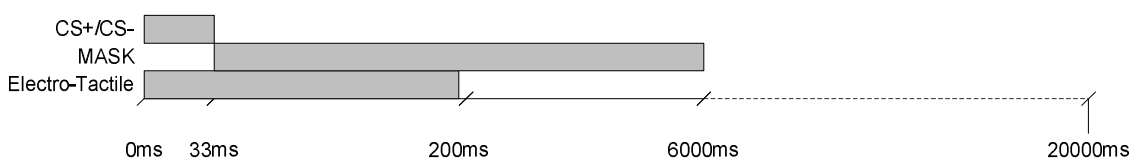

Mask

NoETS

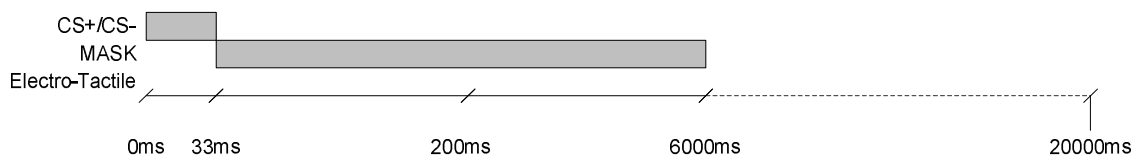

NoMask

ETS

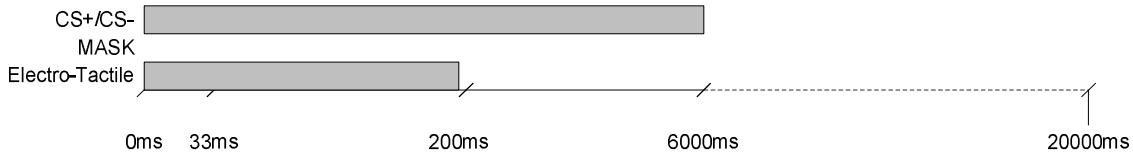

NoMask

NoETS

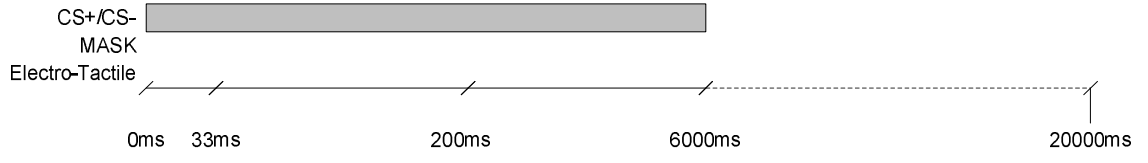

Note ETS = Electro-Tactile Stimulation group, Mask = Masked presentation group. 
IAT and Pavlovian Conditioning 34

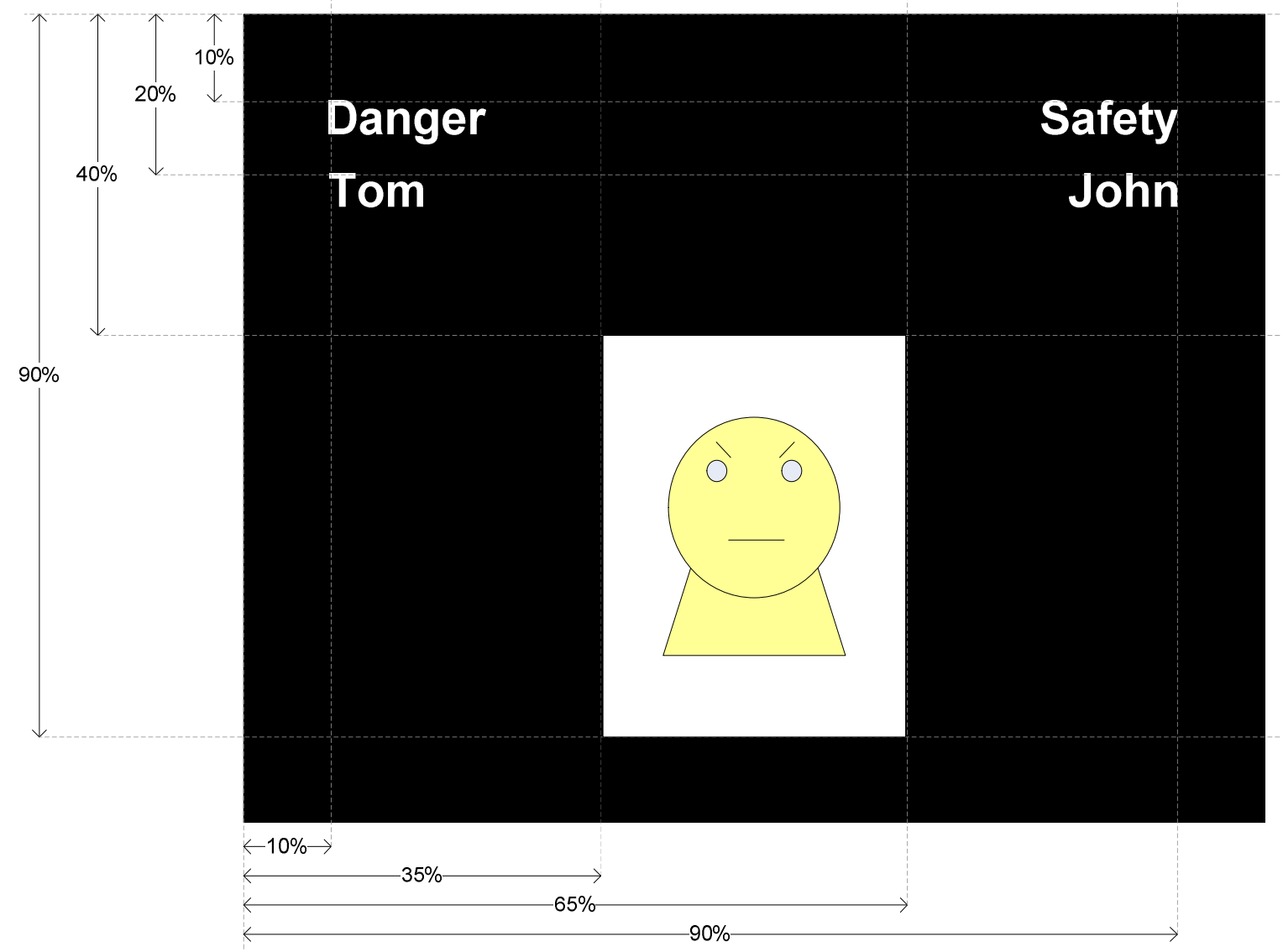




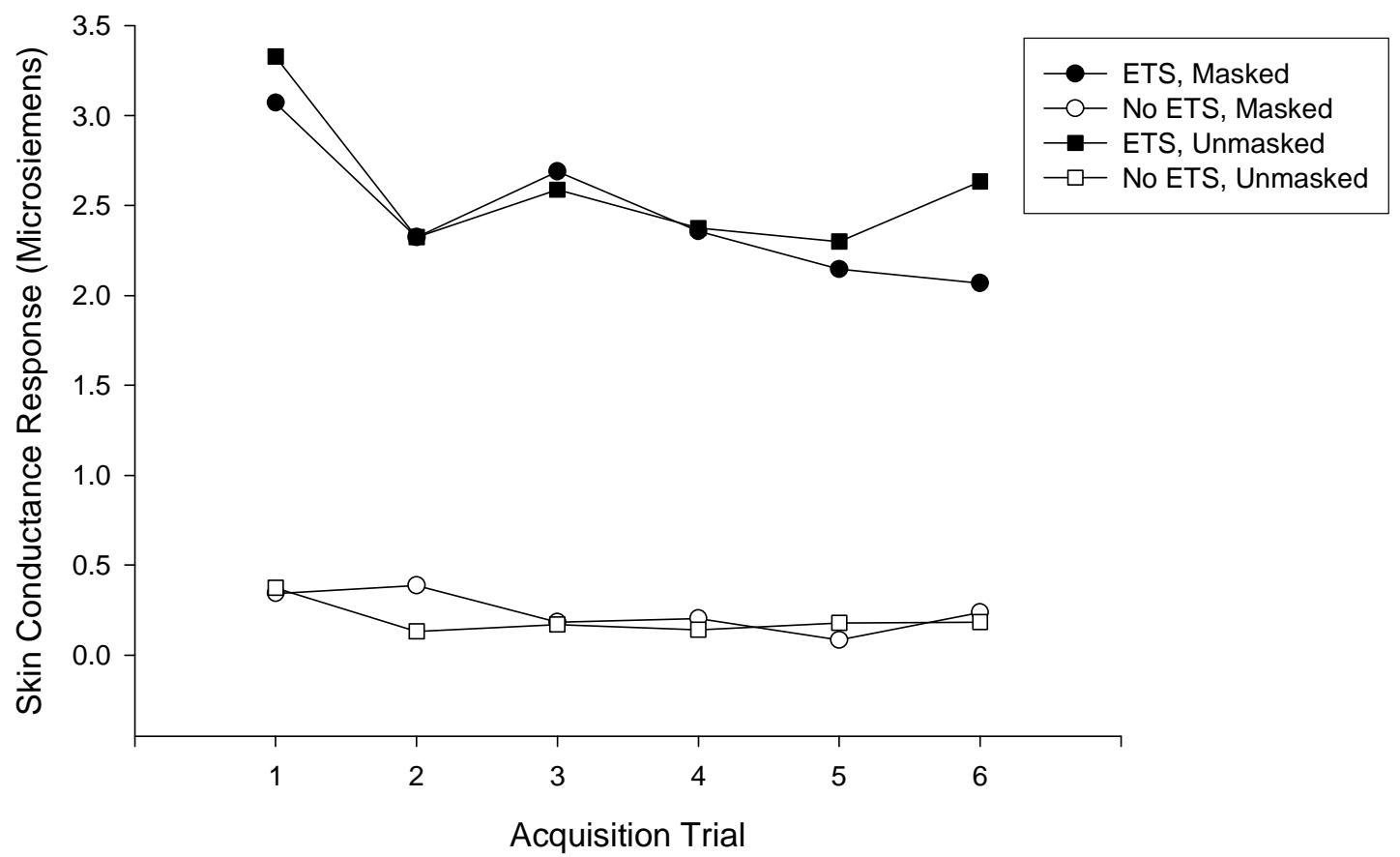




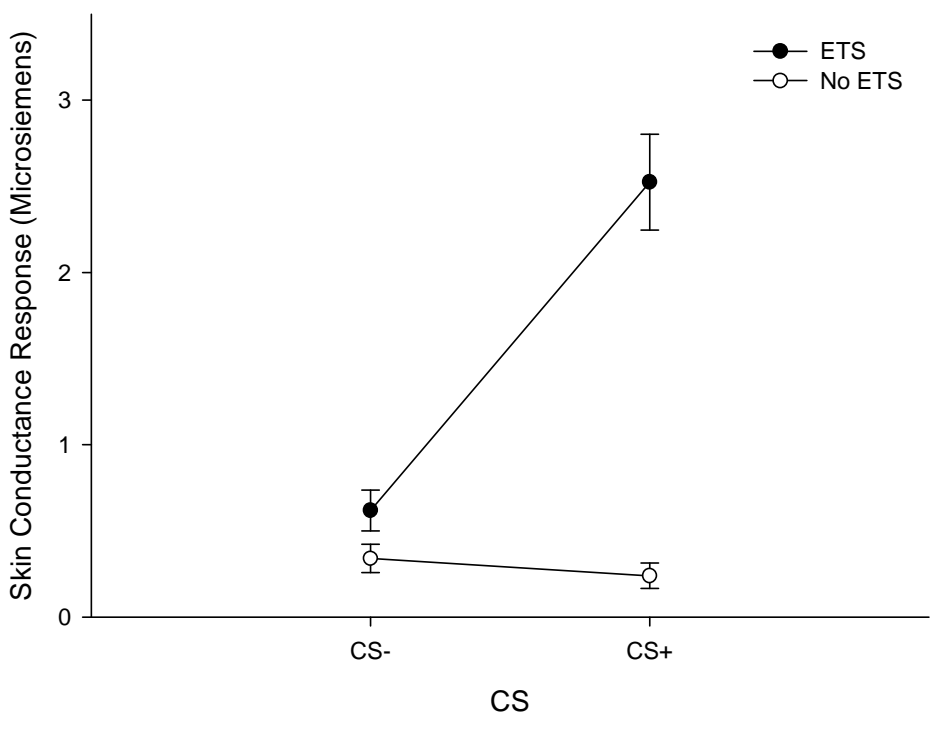

Masked Presentation

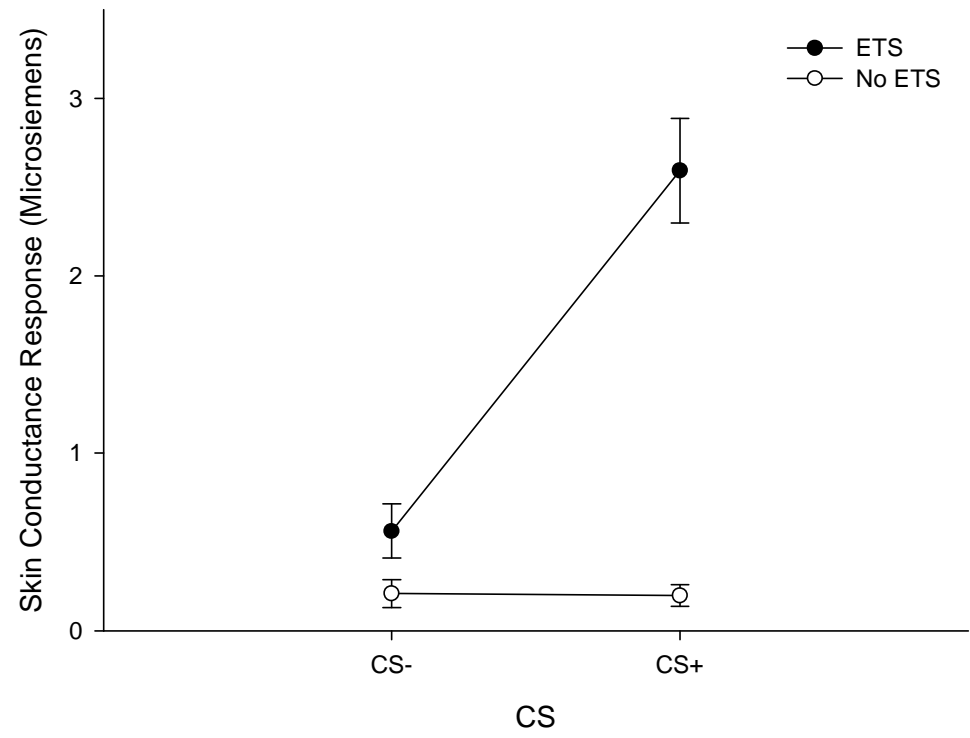

Unmasked Presentation 
IAT and Pavlovian Conditioning 37

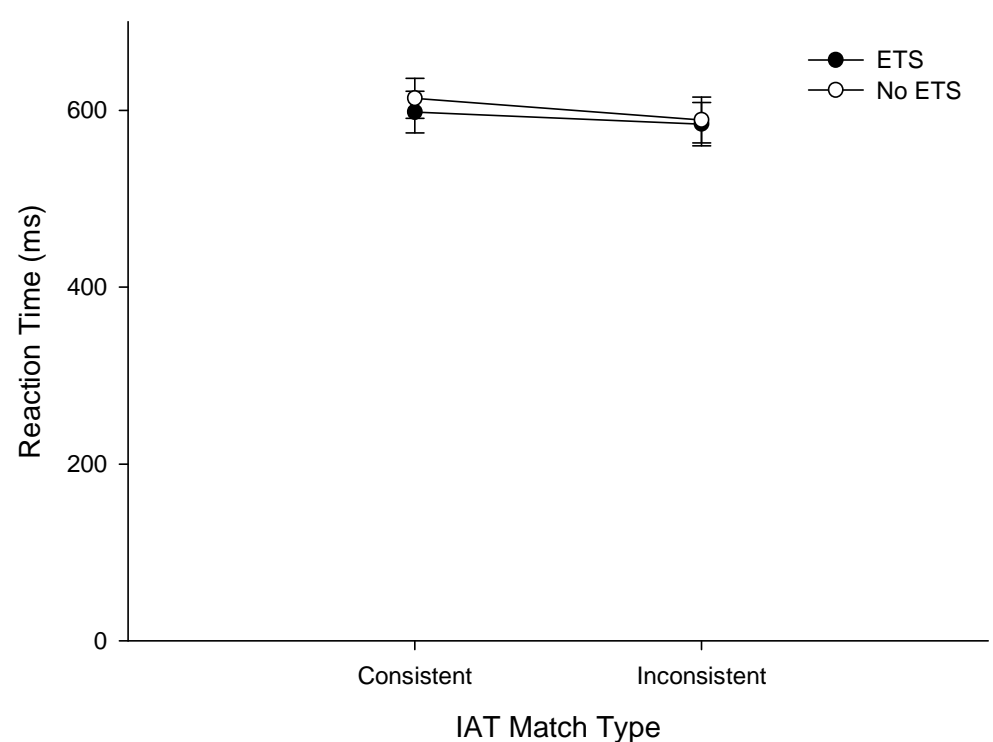

Masked Presentation

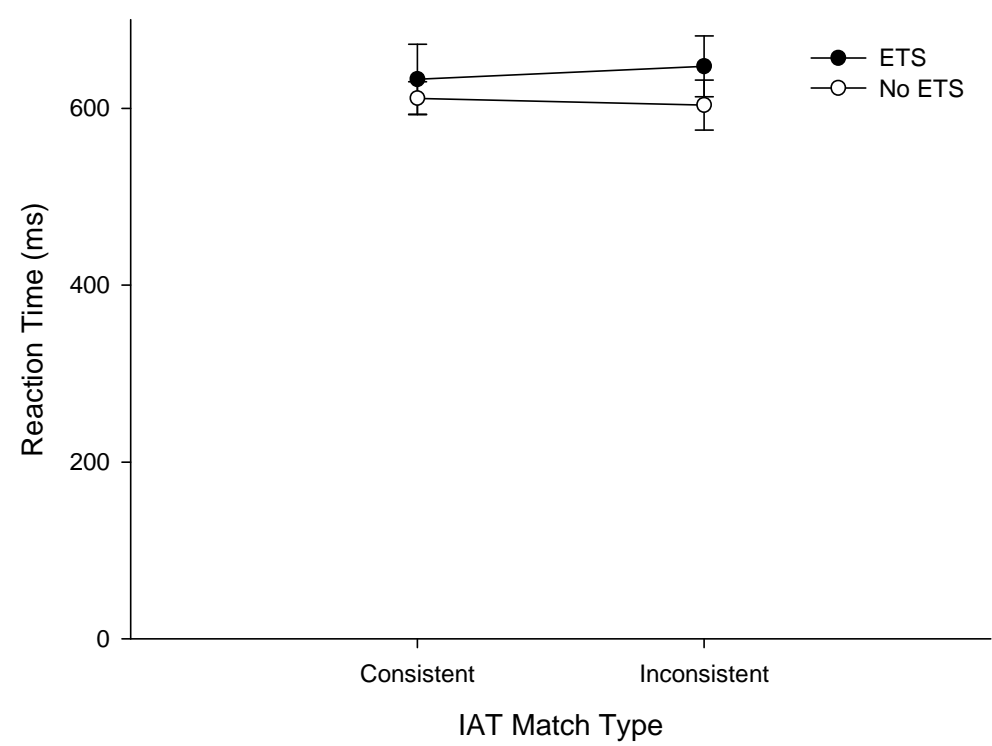

Unmasked Presentation 